\title{
Un panzerotto in bocca italiana!
}

\author{
Miriam Di Carlo
}

PUBBLICATO: 24 MAGGIO 2019

\section{Quesito:}

Alcuni lettori ci chiedono che cosa si indichi con panzerotto, parola diffusa in Italia con significati differenti.

\section{Un panzerotto in bocca italiana!}

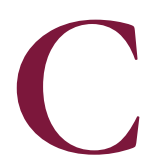

omunemente il panzerotto, o panzarotto nella forma non toscanizzata, è conosciuto in buona parte d'Italia come una 'sorta di grosso raviolo fritto, spec. a forma di mezzaluna, ripieno di un impasto di mozzarella, ricotta e prosciutto, o se dolce, di marmellata' (GRADIT). La parola panzerotto è datata I797 nel GRADIT e nello Zingarelli zorg sulla base del più antico esempio riportato nel GDLI:

Ripiegateci sopra [al composto] la pasta, scaldatela bene e tagliatela collo sperone, a guisa di piccioli ravioli a mezza luna. Nel momento di servire fateli friggere nello strutto ben caldo e serviteli subito di bel colore (Francesco Leonardi, Apicio Moderno, Roma, Gionchi Editore, 1797).

In realtà, però, il brano citato indica il piatto cui siamo soliti fare riferimento, ma non lo nomina.

Il termine si trova invece nelle attestazioni successive, risalenti all'Ottocento, che descrivono grosso modo la stessa ricetta ovvero un raviolo di pasta, a volte lievitata, altre volte sfoglia e simili, farcito con diversi ripieni e fritto in olio o strutto:

Provature in panzarotti alla Napoletana in diverse maniere. Orduvre. Tritarete assai fine le provature, ed uniteci insieme un poco di formaggio parmegiano, o cavallo, o sbrinzo, o altro buon formaggio, un pezzo di butirro, poco sale, pepe pesto, noce moscata, e tre uova crude. Tirate una sfoglia sottile di pasta brisè, o mezza sfoglia, tagliatela in tanti tondi con un taglia pasta, ponete sopra ciascun fondo un poco della suddetta composizione, bagnate all'intorno la pasta con uovo sbattuto, ripiegate in mezzo ad uso di raviolo, e saldate bene all'intorno, oppure fateli in forma tonda coperti con altrettanti tondi della suddetta pasta, fateli friggere nello strutto e serviteli subito. Nel ripieno di questi panzarotti potrete mescolare colla provatura un poco di prosciutto cotto per un momento in una cazzarola con un pezzo di butirro, e poi ben tritato; oppure invece del prosciutto qualche alice dissalata, e spinata, e se vorrete ancor, potrete mettervi un poco di petrosemolo trito. Molte volte ancora inseriscono nel ripieno dei panzarotti un poco di ricotta (Vincenzo Agnoletti, La nuova cucina economica in cui sinsegna la piu facile e precisa maniera di imbandire con raffinato gusto ed economia qualunque delicata mensa [...], Milano, Stamperia Pietro Agnelli in Santa Margarita, I8I9, pp. 32-33);

Panzerotti con mozzarella. Farai la pasta pei panzerotti [...], la levigherai sottilmente col laganatojo polverizzando la tela col fior di farina; farai per questa proporzione un battuto di sei ovi interi, ci porrai once sei di provola grattugiata, ed once sei di mozzarella triturata, ne farai una farsa con pochino di pepe, e di questa ne riempirai li panzerotti attaccando bene gli orli della pasta, e con lo sperone li taglierai, e quindi li friggerai con once 24 di sugna di bel biondo colore, li adatterai nel piatto proprio con salvietta al di sotto (Ippolito Cavalcanti, La cucina teorico-pratica, ovvero Il pranzo periodico di otto piatti al giorno cumulativamente col suo corrispondente riposto [...], Napoli, Stamperia e cartiere del Fibreno, I837, p. II3). 
La prima e l'ultima citazione sono tratte da due opere di autori, il primo (Francesco Leonardi) romano ma formatosi a Napoli, il secondo napoletano (Ippolito Cavalcanti, che si rifa in parte al suo predecessore), mentre la seconda è tratta dal libro di Vincenzo Agnoletti, autore romano che parla esplicitamente di panzarotti alla Napoletana. Si tratta dunque di una preparazione che sembra provenire dal Sud, e infatti il termine associato a questa ricetta viene registrato dal GRADIT e da alcuni dizionari contemporanei (Garzanti 2017, Zingarelli 2019) come parola di origine meridionale, a volte restringendo la nascita del piatto e della parola alla sola area pugliese, anziché a quella napoletana. Il Sabatini-Coletti 2008 pone in appendice al significato che abbiamo segnalato una nuova accezione: 'anche tipo di pasta fresca ripiena a forma di mezzaluna', riconducendo l'origine della parola al Meridione. Infine il Devoto-Oli 20I8, seppur inserendo nell'etimo "voce di area meridionale", aggiunge un secondo significato attribuito al Settentrione:

I. grosso raviolo di pasta tipico pugliese e campano a forma di mezzaluna, ripieno di vari ingredienti (ricotta, mozzarella, prosciutto, salsiccia, o salame, uova, pomodoro) o anche marmellata, fritto in olio bollente;

2. nel Nord Italia, pasta fresca ripiena, di forma analoga.

Sembrerebbe che questo secondo significato si sia avuto per estensione del primo, in relazione sia alla forma di mezzaluna del panzerotto e sia al fatto che si tratta di un raviolo di pasta ripiena. Infatti anche nel ricettario di Ippolito Cavalcanti la forma del panzerotto veniva presa come esempio per modellare paste fresche ripiene:

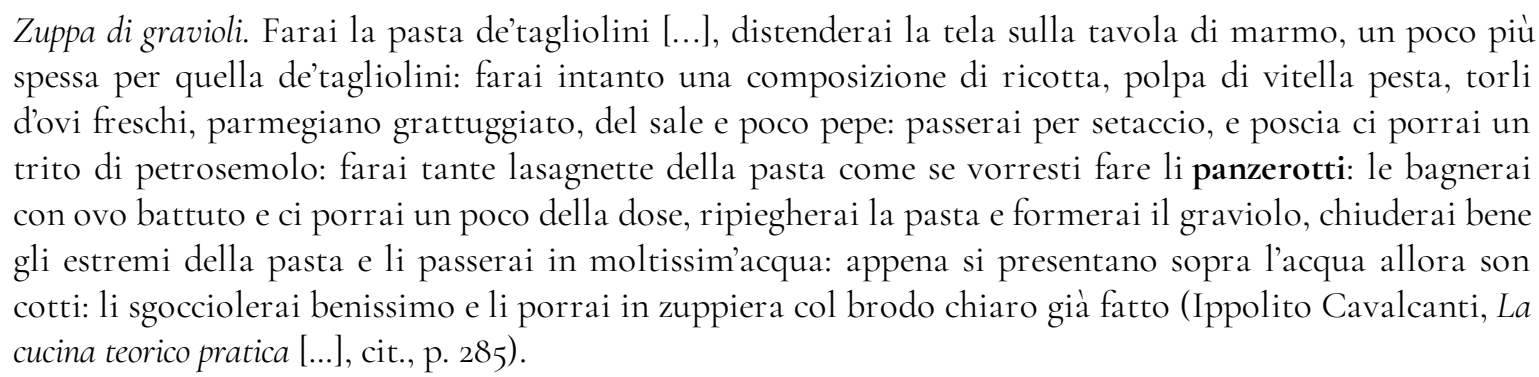

Ma oltre a questo processo analogico che prevede un'estensione del significato primario partendo dalla forma del panzerotto fritto e passando poi per similarità a designare un formato di pasta fresca ripiena tanto al Nord quanto al Sud, possiamo ipotizzare che la parola panzerotto si possa essere formata autonomamente in altre parti d'Italia, riferita ad altri tipi di ricette, completamente differenti rispetto a quella cui siamo abituati nella cultura culinaria popolare. Infatti la parola panzerotto è composta dalla base panza, dal suffisso -otto e da un interfisso che unisce le due strutture (-ar passato poi a -er per influsso del toscano). La base etimologica della parola panzerotto è panza, variante di pancia che i dizionari etimologici (DEI, DELI e l'Etimologico) all'unanimità riconducono al latino păntice $(m)$ 'intestino, ventre'. A lungo i dizionari sono stati concordi nel ricondurre la parola panzerotto a un'origine meridionale, considerando panza variante esclusivamente meridionale di pancia ed -erotto un suffisso tipico meridionale (GRADIT). In realtà la forma panza non è solo tipica dei dialetti meridionali ma è ben presente anche in alcuni dialetti settentrionali (Trentino, Friuli, Liguria: cfr. AIS c. I28 'il ventre'; c. 677 'il mal di ventre'). Inoltre il suffisso -erotto non è tipico del solo Meridione ma concorre alla formazione di parole in italiano: (cfr. Rohlfs I969, p. 364, §Io34 e p. 456, §II43). Dunque la parola panzerotto potrebbe essere una parola dall'origine tanto meridionale quanto settentrionale.

L'uso estensivo del termine panzerotto, usato per designare anche un qualsiasi altro tipo di pasta fresca ripiena che presenti la forma tipica di mezzaluna, richiama tanto il panzerotto fritto, quanto anche il 
cosiddetto pansotto ligure ovvero un tipo di raviolo fresco ripiegato o a forma di triangolo o di mezzaluna, farcito con formaggio fresco e erbe aromatiche, condito di solito con salsa alle noci. Il termine pansotto, simile ma non uguale a panzerotto, sembrerebbe avere unorigine tutta ligure: dalla base pansa, variante ligure di pancia, e dal suffisso -otto (in questo caso si noti l'assenza dell'interfisso $-e r)$.

Come si era già accennato, dato che la base panza può essere sia meridionale che settentrionale e che formazioni di questo tipo per indicare piatti di cucina non sono poi cosi infrequenti, la parola panzerotto potrebbe aver avuto anche un'origine settentrionale, parallela a quella meridionale. Infatti il panzerotto indica a Piacenza e in aree limitrofe, un tipo di pasta ripiena, la cui forma non riprende però quella di mezzaluna. Anzitutto per i panzerotti piacentini, considerati un piatto tipico delle feste, si parte da una base semiliquida, che viene cotta a mo' di crespelle. Ogni crespella viene riempita con un composto di ricotta e spinaci, poi viene tagliata a rondelle di circa tre centimetri e adagiata in una teglia da forno con la parte del condimento a vista. In questo caso la forma del panzerotto è quella di una sorta di grande rosa farcita che può essere arricchita con ragù o besciamella. Sia nella ricetta più nota che in questa piacentina, cosi come nei pansotti liguri, la base panza (e pansa) porta con sé i significati di 'pingue', 'panciuto', 'rigonfio' per la forma dei referenti (tant'e che in Toscana i panzerotti meridionali vengono detti sgonfiotti), e rimanda anche all'idea di 'sazietà' (pancia piena, pancia mia fatti capanna).

A questo punto non dovrebbe sorprendere se al Meridione il termine panzerotto designi anche un altro tipo di piatto, completamente differente rispetto a quello più diffuso e conosciuto. Si potrebbe ipotizzare che questa nuova associazione sia del tutto indipendente da quella comunemente diffusa e che dunque la parola panzerotto, associata a queste altre pietanze, abbia avuto origine autonoma. Ma non si può escludere neanche, viste alcune affinità di tali pietanze con la ricetta del panzerotto (ad esempio la frittura), che questi nuovi significati siano nati per associazione analogica con quello maggiormente diffuso. Confrontando i dizionari dialettali pugliesi (soprattutto di area salentinoleccese) e campani nonché attraverso alcune ricerche sui quotidiani, appare così che il panzerotto può anche essere una sorta di crocchetta fritta di patate o di riso.

Si vedano le seguenti definizioni tratte da diversi dizionari dialettali:

\section{PUGLIA:}

panzarottu s.m. panzarotto, crocchè, bocconcino di patate lesse e riso ridotte a purè, avvoltolato nel pane grattugiato e poi fritto in olio bollente (Antonio Garrisi, Dizionario leccese-italiano, 2 voll., Cavallino, Capone, 1990);

panzaròttu/panzeròttu s.m. crocchetta di patate o riso (Lina Cavallo Conversano, Le récule e lle paròle. Grammatica e dizionario del vernacolo di Sandonaci (parlata leccese), Galatina, Congedo, 2012);

panzarottu agg. e s. m. obeso; crocchetta di carne, patate, riso o altro (Giovan Battista Mancarella, Paola Parlangeli, Pietro Salamac, Dizionario dialettale del Salento, 2 voll., Lecce, Edizioni Grifo, 2013);

panzaróttu m. sg. crocchette di patate (Rosanna Bove, Antonio Romano, Vocabolario del dialetto di Galatone, Lecce, Grifo, 2014). 


\section{CAMPANIA:}

panzaròtto s.m. frittella fatta con pasta di patate e farina con ripieno di vario genere (Francesco D'Ascoli, Alessandro Cutolo, Dizionario etimologico napoletano, Napoli, Edizioni del Delfino, I979);

panzaròtto s.m. crocchetta di forma bislunga fatta con patate pestate, farina, uova, formaggio grattugiato e pepe, con ripieno di mozzarella e fritta a fuoco lento (Antonio Santella, Dizionario etimologico napoletano di provincia. Voci piu in uso raccolte dal vivo parlare, Avellino, Melito, I989);

panzarotto: frittella con ripieno, crocchetta di patate ripieno di salame, mozzarella o prosciutto (Federico Antonio, Capriamoci: indagine riguardante il linguaggio gergale e dialettale degli ultimi pescatori, contadini e cacciatori dell'isole di Capri, s.i., Autorinediti, 2008);

panzarotto [...] - s. m. crocchetta di patate, frittella ripiena (Sergio Zazzera, Dizionario napoletano: il napoletano non è un dialetto: è una lingua, una musica, un modo di essere, Roma, Newton\&Compton Editori, $2013)$.

Anche in un articolo de "la Repubblica" si precisa che il/la crocchè (anche nella variante crucché) 'crocchetta di patate' può essere pure chiamato panzerotto:

Il crocchè, spesso chiamato anche panzerotto, deve il suo nome alla parola francese "croquette". [...] La tipica ricetta napoletana si compone di un impasto di patate, uova, formaggio e pepe, a cui si aggiunge nella versione domestica anche una farcitura di fior di latte e salumi. ([s.f.], La tradizione del crocché si reinventa, Repubblica.it, 24/5/2018).

Potremmo dunque pensare che la forma rigonfia della crocchetta di patate abbia ispirato l'associazione della parola panzerotto a questo referente, solitamente indicato con una parola di origine francese: crocchetta in italiano e crocché in napoletano. Rimane circoscritta alla sola area napoletana e pugliese questa associazione tra la parola panzerotto e la 'polpetta di patate o di riso' che invece, nel resto d'Italia, già prima dell'Artusi, viene identificata dalla parola crocchetta.

Anche in altre parti d'Italia la parola panzerotto designa altre tipologie di pietanze: ad esempio in Sicilia il panzerotto, più spesso al femminile panzerotta, è un dolce a forma di bombetta o brioche siciliana (col classico tuppo ma un po' più piccolo), fatto di farina, zucchero, burro o strutto, latte e farcita, a seconda della tradizione culinaria locale, con crema pasticcera, ricotta, cioccolato ecc.. Riassumendo, la parola panzerotto può indicare:

I. raviolo di pasta lievitata (o sfoglie e simili) ripieno e fritto, solitamente a forma di mezzaluna,

2. tipo di pasta fresca ripiena, di solito a forma di mezzaluna;

3. crespelle farcite, tagliate a rondelle di $3 / 4 \mathrm{~cm}$ e adagiate in teglia, tipiche del Piacentino;

4. crocchette di patate o riso fritte, a volte ripiene (nell'area pugliese e nel Napoletano);

5. (anche al femminile panzarotta), dolce siciliano simile a un bombolotto farcito.

Il significato I, semplificato nel ripieno (pomodoro, mozzarella e prosciutto cotto) rispetto al referente descritto dal GRADIT, a volte può essere indicato con calzone, parola che il GDLI associa a una pietanza assai simile al panzerotto: 
calzone $[. .$.$] 4. Specie di raviolo, agnolotto fatta in casa farcito con pomodoro, cipolle sgombri, baccalà e$ vari ortaggi, che viene fritto in olio e servito caldo (e può essere anche di pasta frolla e dolce).

Di seguito le altre definizioni rilevate nei dizionari a noi più contemporanei:

GRADIT: [1634, cfr. napol. cazone, propr. 'pantalone'] disco di pasta di pane ripiegato in metà e farcito con mozzarella, pomodoro, prosciutto e sim., da mangiarsi cotto al forno o fritto: c. napoletano, alla napoletana || TS. Nella cucina pugliese, specialità salata a base di pasta di pane farcita con pesce, ortaggi e sim., oppure dolce a base di pasta frolla, fichi, mandorle e cioccolata.

Sabatini-Coletti: gastr. Involucro di pasta salata variamente ripieno.

Garzanti 2017: (gastr.) involucro di pasta di pane ripieno di mozzarella, prosciutto ecc., che viene fritto o cotto in forno.

Devoto-Oli 20ı8: GASTRON. Specialità della cucina meridionale, costituita da un disco sottile di pasta lievitata ripiegato in due e contenente mozzarella, prosciutto o altri ingredienti; viene cotto in forno o fritto in padella. || Piatto tipico pugliese di pasta fatta in casa ripiena di acciughe, pomodoro, cipolla, sgombri e altri ingredienti cotto in forno o fritto in padella; puo essere anche dolce, farcito di fichi, mandorle e cioccolato.

Zingarelli 2019: disco di pasta da pizza, farcito con mozzarella, prosciutto, pomodoro e ingredienti diversi secondo le regioni, ripiegato a metà e cotto al forno o fritto.

I 1 calzone, oltre a designare la pizza farcita pugliese, salata o dolce che sia, può riferirsi anche al significato che di solito si associa alla parola panzerotto, con le sole differenze che, rispetto a quest'ultimo, spesso non si ha una forma predefinita (come quella di mezzaluna) e ammette anche la cottura al forno. Secondo altre versioni, il binomio calzone/panzerotto non si risolve in cottura al forno/frittura ma nella grandezza dei referenti: il calzone di solito è più grande rispetto al panzerotto, che invece si può mangiare in un paio di bocconi. Comunemente, e soprattutto fuor di Campania, Puglia eccezion fatta per Piacenza, panzerotto e calzone stanno ormai diventando sinonimi:

Panzerotti (calzoni) fritti. I panzerotti fritti, anche detti calzoni, sono delle squisite frittelle di pasta lievitata, a forma di mezzaluna, ripiene con mozzarella, pomodoro e origano. I panzerotti sono diffusi in tutto il Sud Italia, dal Lazio alla Sicilia, ma i panzerotti più buoni si preparano in Campania e in Puglia: nei paesi di queste regioni sono un cibo da strada, fritto al momento dalle numerose rosticcerie, un appetitoso street food da gustare a qualsiasi ora!

(Redazione Giallo Zafferano, Panzerotti (calzoni) fritti, ricette.giallozafferano.it).

Infine il significato I del panzerotto può essere riferito anche ad altri lessemi: pizza fritta a Napoli, la fritta a Brindisi. Si rilevano poi similarità anche con altri prodotti italiani: i pituni o piduni messinesi (ripieni di scarola, pomodori, acciughe, fritti e a forma di mezzaluna) o gli sgabei della Lunigiana (farciti con salumi, fritti e dalla forma di cilindro o mezzaluna).

Nota bibliografica:

- Rocco Luigi Nichil, Il panzerotto. Dal Meridione con sapore, in Massimo Arcangeli (a cura di), Peccati di lingua. Le 100 parole italiane del Gusto,Soveria Mannelli, Il Rubettino, 2015, pp. 207-210.

- Angelico Prati, Vicende di parole, in "Italia Dialettale", XIII, I937, pp. 77-I25. 


\section{Cita come:}

Miriam Di Carlo, Un panzerotto in bocca italiana! , "Italiano digitale", 2019, IX, 2019/2 (aprilegiugno)

DOI: $10.35948 / 2532-9006 / 2020.3118$

Copyright 2019 Accademia della Crusca

Pubblicato con licenza creative commons CC BY-NC-ND 